\title{
VIRULENCE OF BORDETELLA BRONCHISEPTICA IN THE PORCINE RESPIRATORY TRACT
}

\author{
Louise A. Collings* and J. M. Rutter \\ Agricultural and Food Research Council, Institute for Research on Animal Diseases, \\ Compton, Newbury, Berkshire RG16 ONN
}

\begin{abstract}
SummaRy. The virulence of Bordetella bronchiseptica in gnotobiotic piglets was studied by intranasal infection with 11 cultures derived from eight strains isolated from pigs (4), dogs (2), a human subject and a monkey. Six of the cultures contained organisms in phase I and five contained phenotypically different phase-III or -IV organisms. Of the phase-III and -IV cultures, four were derived from strains that had been isolated in phase I. Colonisation of the nasal cavity was investigated by counting bacteria in nasal swabs and washings. The toxigenicity of cell extracts from each strain and variant was determined by tests of lethality in mice or of cytopathogenicity in cell cultures. The results showed that two phase-I cultures from pigs colonised the nasal cavity and respiratory tract of gnotobiotic piglets better than did four phase-I cultures from other species. Phase-I organisms invariably produced capsules, fimbriae and mannose-resistant haemagglutination of guinea-pig erythrocytes. Four of five cultures in phases III and IV consisted of organisms that did not produce capsules, fimbriae or haemagglutination and colonised the nasal cavity poorly. Phase variation from I to III occurred in culture and in vivo, but variation from III to I occurred in vivo only and was accompanied by enhanced colonisation. Gnotobiotic piglets infected with porcine phase-I organisms exhibited atrophy of the nasal turbinate bones after 28 days; these organisms produced significantly more toxin than did bacteria in phase I from other species, or those in phases III and IV. It was concluded that the development of turbinate atrophy was associated with (1) the ability to produce heavy, persistent colonisation in the nasal cavity, and (2) the production of a heat-labile toxin. Only the two porcine phase-I cultures possessed both properties.
\end{abstract}

\section{INTRODUCTION}

Bordetella bronchiseptica has long been recognised as a pathogen of laboratory and domestic animals (Switzer, 1981). In specific pathogen-free and gnotobiotic pigs, it causes atrophy of the nasal turbinate bones (Ross, Duncan and Switzer, 1963; Shimizu et al., 1971; Kemeny, 1972; Brassinne, Dewaele and Gouffaux, 1976; Rutter, Francis

Received 30 May 1984; accepted 18 Jul. 1984.

* Present address: Royal Free Hospital School of Medicine, Hampstead, London NW3. 
and Sansom, 1982) and, together with Pasteurella multocida (Rutter, 1983), is one of the micro-organisms associated with the complex aetiology of atrophic rhinitis. $B$. bronchiseptica is present in the respiratory tract of virtually all species of animal and is widely distributed in the pig population of most countries (Switzer, 1981). In the United Kingdom, the severity of atrophic rhinitis varies and the prevalence of severe disease is less widespread than is infection with B. bronchiseptica (Giles et al., 1980; Rutter, 1981). This discrepancy may be partly attributable to differences in the virulence of the organism. Ross, Switzer and Duncan (1967) showed that isolates from different animal species varied in their pathogenicity for pigs, and Miniats and Johnson (1980) and Skelly et al. (1980) reported differences in the virulence of porcine isolates of B. bronchiseptica. However, Rutter et al. (1982) were unable to detect significant differences in the virulence of $B$. bronchiseptica from herds with or without clinical atrophic rhinitis.

There are many similarities between $B$. bronchiseptica and the causal organism of whooping cough in man, B. pertussis. Both can occur in one of several phases (Leslie and Gardner, 1931; Nakase, 1957a; Ezzell et al., 1981) and the importance of using "smooth" or phase-I variants for studies of virulence in laboratory animals has been emphasised. Furthermore, disrupted cell preparations of B. pertussis and B. bronchiseptica contain a heat-labile toxin that is lethal for mice (Munoz, 1971; Morse, 1976). In pigs, Hanada et al. (1979) showed that turbinate atrophy could be produced by the repeated intranasal instillation of cell-free material prepared from a phase-I strain of $B$. bronchiseptica by ultrasonic disintegration. However, the relevance of phase and toxigenicity to the virulence of $B$. bronchiseptica for pigs has not been adequately studied. The purpose of the work reported here was to investigate these aspects, with particular reference to colonisation of the nasal cavity and its relation to turbinate atrophy.

\section{MATERIALS AND METHODS}

Piglets. Thirty-five germ-free piglets from five litters were used (table). They were delivered by hysterotomy and reared in pairs in plastic isolators (Rutter et al., 1982).

Bacterial strains and phase variants. The 11 cultures used (table) were derived from eight strains. Six of the cultures were in phase I, three in phase III, and two in phase IV. Strains ASI and GF8 were isolated from two herds with clinical atrophic rhinitis. Strain DCH17 was kindly supplied by Dr L. Irons (Centre for Applied Microbiology and Research, Porton) and strain 57106 by Dr I. McCandlish (Glasgow University Veterinary School). Strains 10540 and 452 were obtained from the National Collection of Type Cultures. All strains except no. 452 were obtained in phase I; strain 452 was obtained in phase III. The phase-I variant of strain 452 was isolated from a gnotobiotic piglet infected with the original phase-III culture. Phase-III and -IV variants of the phase-I strains AS1, GF8, LI and FW5 were obtained by repeated subculture in peptone water (Nakase, 1957b). The parent strains, LI and FW5, of the phase-IV variants were isolated in phase I from two herds without clinical atrophic rhinitis. Phases I, III and IV (Nakase, 1957a) were identified on the basis of colonial morphology and the presence of haemolysis after aerobic incubation $\left(48 \mathrm{~h}, 37^{\circ} \mathrm{C}\right)$ on Bordet-Gengou (BG) agar (Difco) containing defibrinated horse blood (Oxoid) 15\% v/v. Phase-II (intermediate) variants were not studied in this investigation because they were unstable on subculture. Stable variants in phases III and IV, consisting of large flat non-haemolytic colonies, were established by repeated subculture of single colonies on BG agar. All cultures were stored lyophilised until just before use.

Infection and sampling procedures. Piglets aged 5-6 days were infected by the intranasal route. Bacterial suspensions were prepared from overnight growth on BG agar plates (Rutter $e t$ $a l ., 1982)$. The inoculum for these plates was taken from a single colony to try to ensure that the 
infecting dose contained organisms in only one phase. Piglets were given $10^{3}, 10^{5}$ and occasionally $10^{9} \mathrm{cfu}$; two control piglets were given saline alone (table). Nasal washings and nasal swabs were taken every 2-3 days and bacterial counts were determined on BG agar (Rutter, 1981) to obtain colonisation profiles. The results were analysed by a 'Genstat' programme (Rothamsted Experimental Station, 1977) with a "split plot in time" analysis; differences greater than $10^{0.4} \mathrm{cfu} / \mathrm{g}$ were significant $(\mathrm{p}<0.05)$. Blood samples were taken from the anterior vena cava at weekly intervals after infection; the serum was separated and agglutinins to suspensions of homologous organisms were titrated by a microtitration method (Jenkins, 1978).

Necropsy, histology and analytical procedures. Piglets were killed after 28-30 days by intravenous injection of sodium pentobarbitone (Euthatal; May and Baker). Macroscopic signs of disease were noted and turbinate atrophy was assessed (Done, Richardson and Hebert, 1964) after sectioning the upper jaw at the first cheek tooth with a band saw. Small sections of the upper jaw were fixed and the dimensions of the turbinate bones were measured (Rutter et al., 1982). Swabs from the nasal cavity, pharynx, trachea, spleen, kidney and heart blood were cultured on $\mathrm{BG}$ agar. The lungs were examined for pneumonic lesions and a portion of the right cardiac lobe was cultured.

Preparation of cell-free extracts. Bacterial suspensions were prepared in $0.05 \mathrm{~m}$ phosphatebuffered saline (PBS, $p \mathrm{H} \mathrm{7.2)}$ by making a ten-fold dilution (w/v) of growth $\left(20 \mathrm{~h}, 37^{\circ} \mathrm{C}\right)$ from $\mathrm{BG}$ agar. The cell suspension $\left(c .10^{11} \mathrm{cfu} / \mathrm{ml}\right)$, in an ice-bath, was disrupted ultrasonically (Sonifier B12; Branson Sonic Power Co., Connecticut, USA) with a Dawe Accoustic Booth (Ultrasonics Ltd, Shipley, UK) at 100-110 W output in six 20-s bursts with intervals for cooling. The resulting opaque suspension was centrifuged $(30000 \mathrm{~g}, 30 \mathrm{~min})$ and the supernate sterilised by filtration through a $0 \cdot 22-\mu \mathrm{m}$ membrane (Millipore). The protein concentrations of the extracts (range $3 \cdot 8-5 \cdot 3 \mathrm{mg} / \mathrm{ml}$ ) were estimated by spectrophotometry (Ehresmann, Imbault and Weil, 1973). The extracts were stored at $-20^{\circ} \mathrm{C}$ and two or three from each strain were examined.

Toxicity tests. Cell-free extracts were tested in an assay of lethality in mice or in a cell-culture system. Groups of 4-5 Compton white mice weighing 15-18 g were given intraperitoneal injections of $0.5 \mathrm{ml}$ of a range of dilutions of the extract. Deaths were recorded up to 7 days and the LD50 values determined by Probit analysis with a "Genstat" programme. In a cell-culture test devised to replace the mouse test, dilutions of the extracts were added to suspensions of embryo bovine lung (EBL) cells (Rutter and Luther, 1984). The cytopathic effect of the $B$. bronchiseptica toxin was determined by microscopic examination of the cell sheets stained by gentian violet $(0.2 \%)$ after $2-3$ days when the control mono-layers had become confluent (Collings, 1983).

Haemagglutinating activity ( $H A$ ) and production of fimbriae. A qualitative tile test (Duguid and Gillies, 1957) or a microhaemagglutination plate test (Jones and Rutter, 1974) with guinea-pig erythrocytes was used to determine the HA of cultures harvested from BG agar (Collings, 1983). To investigate mannose-resistant HA, D-mannose $0.5 \%$ or $2 \% \mathrm{w} / \mathrm{v}$ was added to the diluent. Production of fimbriae was examined in the electronmicroscope by negative staining with phosphotungstic acid $(2 \%, p \mathrm{H} 7.0)$ or gold-palladium metal shadowing techniques (Collings, 1983).

Capsule production. Capsules were identified in smears stained with a modified Wright's stain (Lawson and Charlottesville, 1940).

\section{RESULTS}

\section{Clinical signs}

Some infected piglets sneezed occasionally and had transient nasal discharges but none showed snout malformations.

\section{Colonisation profiles and phase variation}

Bacterial counts of nasal swabs and washings gave comparable results, although washings yielded fewer organisms. Dose had little effect on the colonisation profiles 
TABLE

Properties of B. bronchiseptica strains and variants and results of infection in gnotobiotic piglets

\begin{tabular}{|c|c|c|c|c|c|c|c|c|c|c|c|c|}
\hline \multicolumn{3}{|c|}{ Strain } & & & \multirow{3}{*}{$\begin{array}{l}\text { Haemag- } \\
\text { glutina- } \\
\text { ting } \\
\text { activity }\end{array}$} & \multirow{3}{*}{$\begin{array}{l}\text { Cap- } \\
\text { sule }\end{array}$} & \multirow{3}{*}{$\underset{(\mathrm{cfu})}{\text { Inoculum }}$} & \multirow{3}{*}{$\begin{array}{c}\text { Number } \\
\text { of } \\
\text { piglets }\end{array}$} & \multirow{3}{*}{$\begin{array}{c}\text { Colonisa- } \\
\text { tion } \\
\text { profile }\end{array}$} & \multirow{2}{*}{\multicolumn{2}{|c|}{ Toxicity }} & \multirow{3}{*}{$\begin{array}{c}\text { Turbin- } \\
\text { ate } \\
\text { score }\end{array}$} \\
\hline \multirow[b]{2}{*}{ No. } & \multirow[b]{2}{*}{ Origin } & \multirow{2}{*}{$\begin{array}{c}\text { Phase } \\
\text { when } \\
\text { obtained }\end{array}$} & \multicolumn{2}{|c|}{ Phase of cultures } & & & & & & & & \\
\hline & & & inoculated & recovered & & & & & & titre* & group $\dagger$ & \\
\hline GF8 & Pig & I & I & 1 & + & + & $10^{9}$ & 2 & a & 2400 & 1 & $3 \cdot 1$ \\
\hline AS1 & Pig & 1 & I & I and III & + & + & $\begin{array}{l}105 \\
10^{5}\end{array}$ & 1 & a & 2240 & 1 & $2 \cdot 9$ \\
\hline 10540 & Man & I & I & $\mathrm{I}$ and III & + & + & $\begin{array}{l}10 \\
10^{9}\end{array}$ & $\frac{3}{2}$ & b & 896 & 2 & 1.6 \\
\hline $\begin{array}{l}\text { DCH17 } \\
57106\end{array}$ & $\begin{array}{l}\text { Monkey } \\
\text { Dog }\end{array}$ & I & $\begin{array}{l}\text { I } \\
\text { I }\end{array}$ & $I$ and III & $\begin{array}{l}+ \\
+\end{array}$ & $\begin{array}{l}+ \\
+\end{array}$ & $\begin{array}{l}10^{3} \\
10^{5}\end{array}$ & 2 & $\begin{array}{l}\mathrm{b} \\
\mathrm{b}\end{array}$ & $\begin{array}{r}1440 \\
280\end{array}$ & $\begin{array}{l}2 \\
2\end{array}$ & $\begin{array}{l}1.0 \\
1.0\end{array}$ \\
\hline 452 & Dog & [II & I & I and III & + & + & $\begin{array}{l}10^{5} \\
10^{3}\end{array}$ & $\frac{3}{2}$ & c & 40 & 3 & $1 \cdot 0$ \\
\hline GF8 & Pig & I & III & III & - & + & $\begin{array}{l}10^{5} \\
10^{3}\end{array}$ & 1 & $\mathrm{~b}$ & 25 & 3 & 1.5 \\
\hline ASI & Pig & 1 & III & $I$ and III & $-\rightarrow+$ & $-\rightarrow+$ & $\begin{array}{l}10^{5} \\
10^{3}\end{array}$ & 1 & d & NT & $3 \rightarrow 1$ & $3 \cdot 0$ \\
\hline 452 & Dog & III & III & I and III & $-\rightarrow+$ & $-\rightarrow+$ & $\begin{array}{l}10^{5} \\
10^{3}\end{array}$ & $\frac{1}{2}$ & d & 25 & 3 & 1.0 \\
\hline LI & Pig & I & IV & IV & - & - & $\begin{array}{l}10^{5} \\
10^{3}\end{array}$ & 1 & $\mathrm{e}$ & 25 & 3 & 0.5 \\
\hline FW5 & Pig & I & IV & IV & - & - & $\begin{array}{l}10^{5} \\
10^{3}\end{array}$ & $\begin{array}{l}1 \\
1\end{array}$ & $\mathrm{e}$ & NT & NT & 1.0 \\
\hline$\ldots$ & $\ldots$ & $\ldots$ & $\ldots$ & $\ldots$ & $\ldots$ & $\ldots$ & diluent & 2 & $\ldots$ & $\ldots$ & $\ldots$ & 0.8 \\
\hline
\end{tabular}

$+=$ Present; $-=$ absent; a-e = colonisation profiles (see Results and fig. 1 ); NT $=$ not tested.

$\rightarrow+=$ Negative when inoculated, positive when recovered in phase I;

$3 \rightarrow 1=$ Group 3 when inoculated, group 1 when recovered in phase $\mathbf{I}$.

* Mean dilution that gave cytopathic effect in EBL cells.

$\uparrow 1=$ Highly toxic; $2=$ moderately toxic; $3=$ poorly or non toxic in mice.

$\ddagger$ Mean grade.

except that maximal colonisation was reached more slowly after small than after large doses.

A summary of the results is given in the table. All 11 strains and variants colonised the nasal cavity of germ-free piglets. The two phase-IV variants (strains LI and FW5) colonised poorly (profile e, fig. 1), their counts occasionally exceeding $10^{4} \mathrm{cfu} / \mathrm{g}$ but generally reaching only $10^{2}-10^{3 \cdot 5} \mathrm{cfu} / \mathrm{g}$; phase variation did not occur.

In contrast, two porcine phase-I strains (GF8 and AS1) colonised rapidly from an inoculum of $10^{3} \mathrm{cfu}$; their numbers reached $10^{8} \mathrm{cfu} / \mathrm{g}$ within $3-5$ days (see fig. 1) and persisted at about that level for 4 weeks after infection (profile a). The human (10540), simian (DCH17) and canine (57106) phase-I strains reached numbers similar to those of the porcine phase-I strains during the first 12 days after infection (profile $b$ ) but decreased more rapidly to $10^{4}-10^{5} \mathrm{cfu} / \mathrm{g}$ after 4 weeks. A phase-I variant from a canine phase-III strain (452) colonised less well initially than those from pigs, reaching only $10^{6} \mathrm{cfu} / \mathrm{g}$, decreasing until the 20 th day, and then rising again (profile $\mathrm{c}$ ).

Two of the three phase-III variants (strains AS1 and 452) showed poor early colonisation, reaching only $10^{4} \mathrm{cfu} / \mathrm{g}$; however, phase-I variants were recovered from all of the piglets after a few days (profile $d$ ). The formation of phase-I variants was accompanied by improved nasal colonisation, which in the case of AS1, then resembled profile a. In-vivo phase variation from III to I occurred with the canine strain 452 rapidly (1-4 days after infection), but with the porcine strain AS1 slowly (6-10 days). Two serial passages of the strain 452 phase-I variant in gnotobiotic pigs showed that its colonisation profile (c) was unaltered and no significant turbinate atrophy was detected at necropsy.

Organisms possessing a capsule were invariably in phase I except for the phase-III 


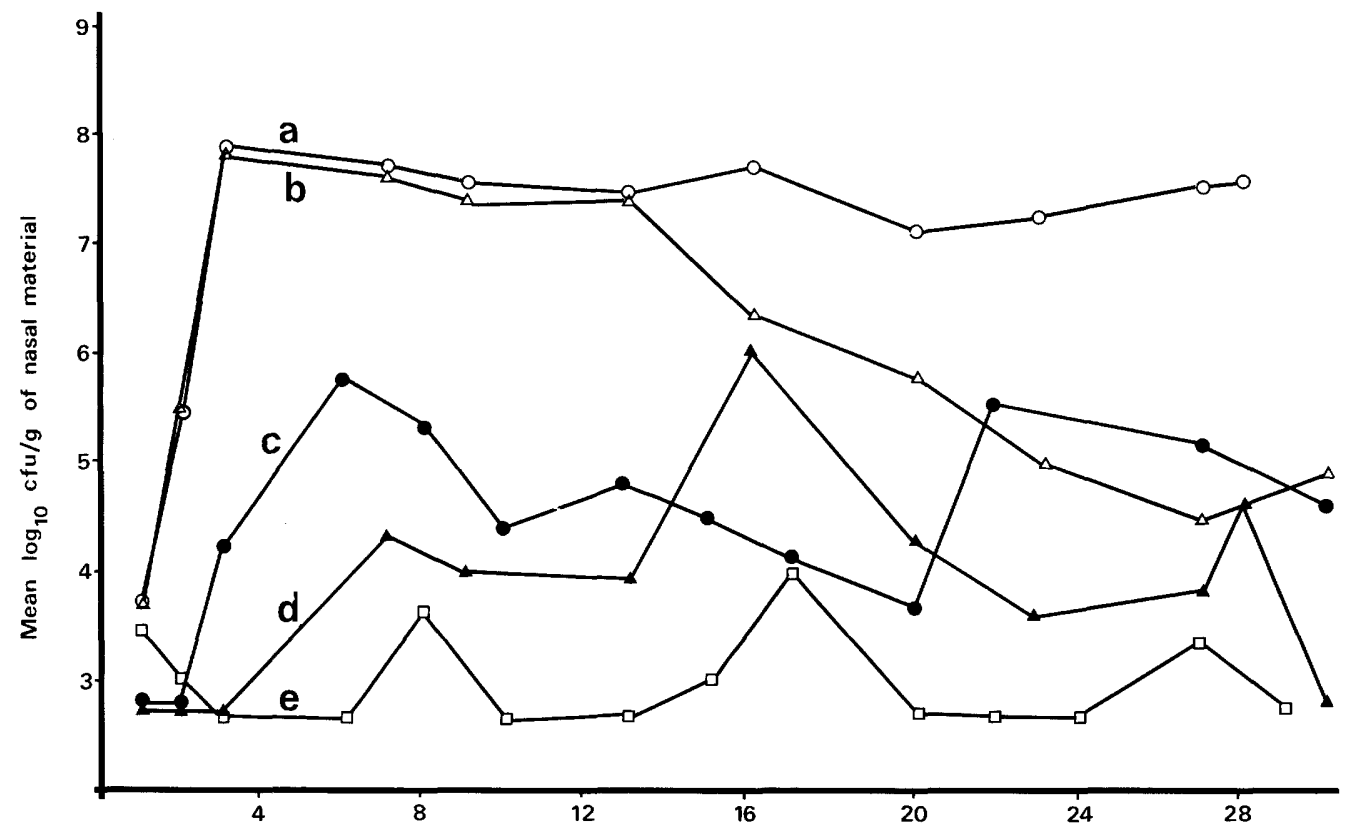

FIG. 1.-Colonisation profiles for piglets infected with different strains and variants of B. bronchiseptica. Differences $>0.4 \log _{10} \mathrm{cfu} / \mathrm{g}$ were significant $(\mathrm{p}<0.05)$. $\mathrm{O}-\mathrm{O}=$ Profile a (phase-I porcine strains AS1 and GF8); $\Delta-\Delta=$ profile b (phase-I non-porcine strains 10540, DCH 17 and 57106; phase-III porcine strain GF8); $-\longrightarrow=$ profile c (phase-I non-porcine strain 452 ); $\Delta-\mathbf{\Delta}=$ profile d (phase-III variants of strains AS1 and 452; the increased counts from the thirteenth day were accompanied by a change to phase I); $\square-\square=$ profile e (phase-IV strains FW5 and LI).

variant of strain GF8. The latter produced good early colonisation (profile b) but did not persist in high numbers or change phase.

\section{Findings at necropsy}

Despite colonisation of the nasal cavity by all of the strains, only the 10 pigs given the porcine phase-I strains (AS1 and GF8) and with the phase-III culture AS1 which reverted to phase I were seen at necropsy to have significant atrophy of the nasal turbinate bones (mean grade $2 \cdot 9-3 \cdot 1$; table). These strains, unlike the others, had penetrated more deeply into the respiratory tract and were isolated from the lungs and lower trachea. Although there was slight shrinkage of the turbinate bones (mean grade $1 \cdot 0-1 \cdot 6)$ in the other piglets, particularly those infected with cultures of strains 10540 , DCH17 and GF8, the reduction in size was not significant.

Phase-III variants were cultured from the nasal cavity of piglets given two of the six phase-I cultures (57106 and 452) and from the pharynx of those given four cultures (AS1, 10540, 57106 and 452). Extensive pneumonia was observed in five of the eight piglets given phase-I pig strains (AS1 and GF8). No isolations were made from the liver, kidney, spleen or heart blood. 


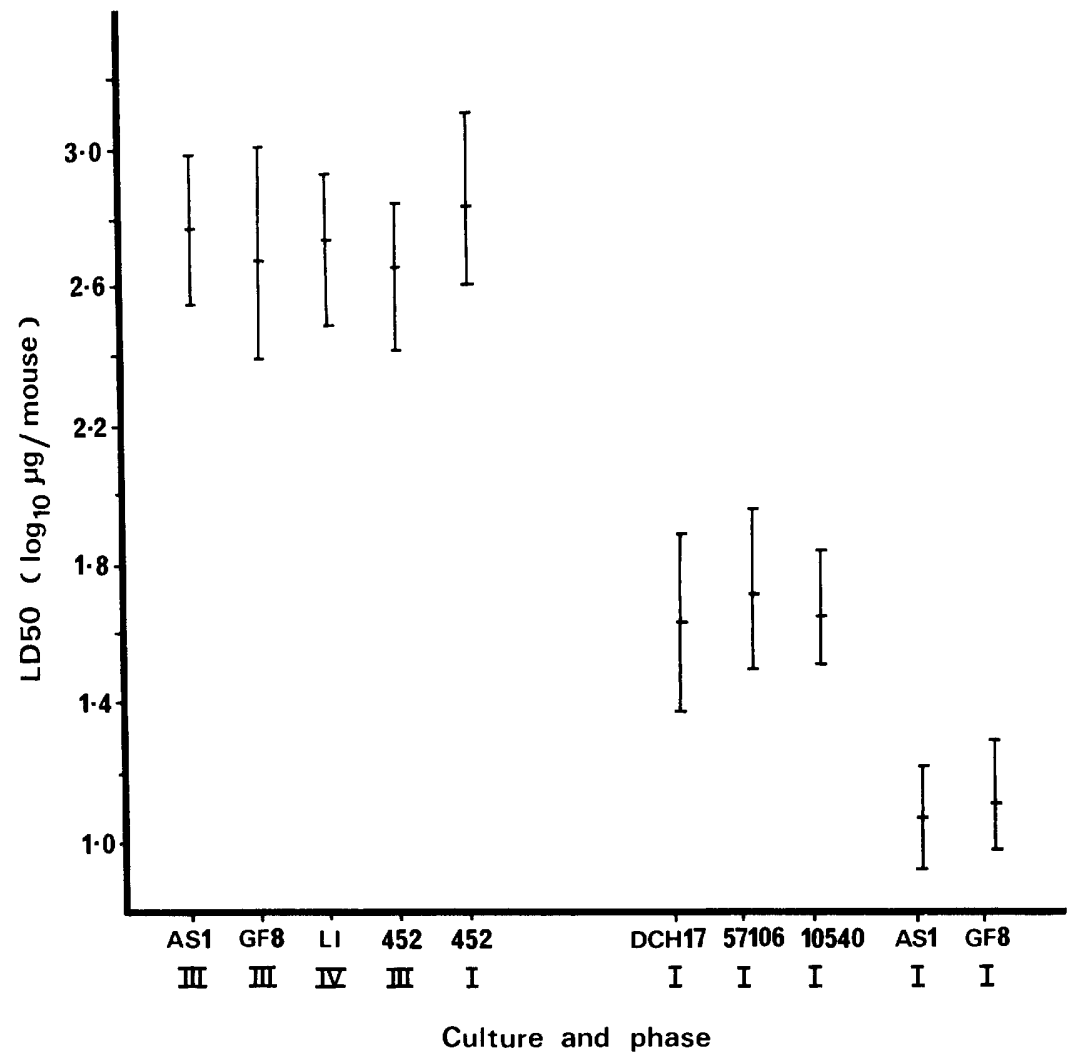

FIG. 2.-LD50 values of extracts of different strains and variants of B. bronchiseptica. (Mean values and $95 \%$ confidence limits.)

\section{Antibody titres}

Serum agglutinins were first detected in piglets 12-14 days after infection in titres of 4-2560. The titres at 28 days were in the range 1024-10240. The phase-IV variants, despite poor colonisation, produced agglutinin titres in the range 2048-8192 at 28 days. The change of phase from III to I in vivo was also reflected in the appearance of high titres of agglutinins to phase-I bacteria by the 28 th day.

\section{Haemagglutinating activity and fimbriae}

All phase-I strains produced mannose-resistant HA in both test procedures and fimbriae after growth on BG agar. With the six phase-I cultures, HA titres varied within the range 64-2048 in different tests. In contrast, HA and fimbriae were not detected in the five phase-III or -IV variants, but were present in the two phase-I revertants (AS1 and 452) recovered from the piglets.

\section{Toxicity of cell-free extracts}

Results in the mouse assay and the cell culture assay were consistent and 
comparable. The strains could be divided into three groups (table, and fig. 2) which differed significantly $(p<0.005)$. Group 1 contained the two porcine phase-I strains, which were highly toxic; group 2 contained three non-porcine phase-I strains, which were moderately toxic; and group 3 contained the phase-I revertant of the canine strain and all the phase-III strains, which were virtually non-toxic.

\section{DisCUSSION}

Colonisation of the respiratory tract and the production of turbinate atrophy by $B$. bronchiseptica is a complex process and these investigations in gnotobiotic piglets have provided a better understanding of the role of bacterial phase and toxigenicity in the pathogenesis of the lesions. The relationship of phase to virulence in guinea-pigs is well documented (Nakase, 1957a). Our results have shown that five of six phase-I cultures were more effective than four of five phase-III or -IV cultures in producing respiratory-tract colonisation of gnotobiotic piglets. Although all of the six phase-I cultures contained organisms that, on BG agar, produced capsules, haemolysin, fimbriae and HA, only the two derived from pigs caused heavy persistent infection in the nasal cavity. This might have been due to quantitative differences in the production of capsules or fimbriae in vivo which we were unable to detect, or to other factors. The possible importance of capsule production was demonstrated by the colonisation profile of the capsulate phase-III variant of strain GF8; this variant was non-fimbriate and did not change to phase I, yet colonised as well as the phase-I variants from species other than the pig.

Phase variation by $B$. bronchiseptica in vitro is well recognised but in-vivo variation has not been studied. Our results indicated that after infection with phase-I cultures, organisms in phase I were generally recovered from the nasal cavity, trachea and lungs, whereas by the 28 th day, phase-III variants were often recovered from the pharynx. This change of phase might have been caused by the production of antibodies to $B$. bronchiseptica; serum agglutinins were demonstrated in all piglets after 12-14 days and the titres increased rapidly up to the 28 th day. In vitro, only a change from phase I to III without reversion was observed. Reversion from III to I in vivo was, therefore, unexpected but was observed with two different phase-III variants. It is unlikely that we were misled by contamination of the inoculum with phase-I organisms; such organisms would have had to have been contained within the single phase-III colonies picked for the numerous sub-cultures used to establish phase stability. The absence of phase reversion in vivo of the GF8 phase-III culture also argues against this explanation. Synthesis of the additional phase-I properties presumably requires a greater expenditure of energy by the micro-organism. In vitro, phase III organisms exhibit a much shorter lag period of growth than those in phase I (Dr A.J. Lax, personal communication) but this did not appear to occur in the porcine nasal cavity with non-capsulate phase-III cultures. The two phase-IV cultures that we studied were similar phenotypically to the three cultures in phase III but were distinguished retrospectively by their inability to revert to phase I in vivo. Phase-IV variants of $B$. pertussis are also deficient in a wide range of cellular components and activities by comparison with phase-I strains (Wardlaw et al., 1976), but the mechanisms controlling the loss of such characters are poorly understood. Since this work was 
completed, Weiss and Falkow (1984) have demonstrated that reversion to phase I can be observed in vitro in $B$. pertussis and have proposed a model for phase variation.

Ross et al. (1967) showed that isolates of $B$. bronchiseptica from a rat and a dog caused virtually no turbinate atrophy in specific pathogen-free pigs. Our results confirmed these findings in gnotobiotic pigs with isolates from a human subject, a monkey and a dog. Whether such strains introduced into pig herds would persist remains to be determined, but in our experiments their virulence was not enhanced by serial passages in gnotobiotic piglets, and turbinate atrophy was produced only by two phase-I strains isolated from pigs. Clearly, colonisation of the nasal cavity of gnotobiotic piglets by $B$. bronchiseptica was not by itself sufficient to produce significant atrophy. Phase-I strains isolated from other species and the capsulate phase-III variant colonised relatively well but produced minimal atrophy. This might be explained by our observation that these strains were less toxigenic than the porcine phase-I strains. Heat-labile toxin from both $B$. bronchiseptica and B. pertussis is predominantly intracellular; it is released by ultrasonic disintegration and, presumably, upon death and lysis of the organism (Morse, 1976). Such material may have been responsible for the turbinate atrophy observed in pigs after repeated intranasal instillation of toxin from a phase-I strain of B. bronchiseptica (Hanada et al., 1979). Although the toxins produced by $B$. bronchiseptica have not been adequately characterised, a number of activities, e.g. adenylate cyclase production, have been identified in $B$. pertussis. Adenylate cyclase production has been identified in some strains of $B$. bronchiseptica (Endoh et al., 1980) and the porcine phase-I strain (AS1) used here produced it, whereas its phase-III variant did not (Dr A.J. Lax, personal communication). Purification of the various active substances produced by phase-I porcine strains of $B$. bronchiseptica would help to elucidate their role in the pathogenesis of turbinate atrophy in pigs.

This study was supported by an A.F.R.C. Research Studentship to LAC. We are indebted to Mr D.C. Davies and Mr M.J. Dennis and their staff for providing and rearing the gnotobiotic piglets.

\section{REFERENCES}

Brassinne M, Dewaele A, Gouffaux M 1976 Intranasal infection with Bordetella bronchiseptica in gnotobiotic piglets. Research in Veterinary Science 20: 162-166.

Collings L A 1983 Pathogenicity of Bordetella bronchiseptica in porcine atrophic rhinitis. Ph.D. Thesis, University of Reading.

Done J T, Richardson M D, Hebert C N 1964 Rhinitis of swine: A survey carried out in Britain in 1956-57. In: Animal Disease Survey No. 3, H.M. Stationery Office, London, p 29.

Duguid J P, Gillies R R 1957 Fimbriae and adhesive properies in dysentery bacilli. Journal of Pathology and Bacteriology 74: 397-411.

Ehresmann B, Imbault P, Weil J H 1973 Spectrophotometric determination of protein concentration in cell extracts containing tRNA's and rRNA's. Analytical Biochemistry 54: 454-463.

Endoh M, Takezawa T, Nakase Y 1980 Adenylate cyclase activity of Bordetella organisms. Its production in liquid medium. Microbiology and Immunology 24: 95-104.

Ezzell J W, Dobrogosz W J, Kloos W E, Manclark C R 1981 Phase-shift markers in Bordetella: alterations in envelope proteins. Journal of Infectious Diseases 143: 562-569.

Giles C J, Smith I M, Baskerville A J, Brothwell E 1980 Clinical, bacteriological and epidemiological observations on infectious atrophic rhinitis of pigs in Southern England. Veterinary Record 106: 25-28. 
Hanada M, Shimoda K, Tomita S, Nakase Y, Nishiyama Y 1979 Production of lesions similar to naturally occurring swine atrophic rhinitis by cell-free sonicated extract of Bordetella bronchiseptica. Japanese Journal of Veterinary Science 41: 1-8.

Jenkins E M 1978 An agglutination test for the detection of Bordetella bronchiseptica infection in swine. Canadian Journal of Comparative Medicine and Veterinary Science 42: 286-292.

Jones G W, Rutter J M 1974 The association of K88 antigen with haemagglutinating activity in porcine strains of Escherichia coli. Journal of General Microbiology 84: 135-144.

Kemeny L J 1972 Experimental atrophic rhinitis produced by a Bordetella bronchiseptica culture in young pigs. Cornell Veterinarian 62: 477-485.

Lawson G M 1940 Modified technique for staining capsules of Haemophilus pertussis. Journal of Laboratory and Clinical Medicine 25: 435-438.

Leslie P H, Gardner A D 1931 The phases of Haemophilus pertussis. Journal of Hygiene 31: 423-434.

Miniats O P, Johnson J A 1980 Experimental atrophic rhinitis in gnotobiotic pigs. Canadian Journal of Comparative Medicine and Veterinary Science 44: 358-365.

Morse S I 1976 Biologically active components and properties of Bordetella pertussis. Advances in Applied Microbiology 20: 9-26.

Munoz J 1971 Protein toxins from Bordetella pertussis. In: Kadis S, Montie T C, Ajl S J (eds) Microbial toxins, vol 2A, Academic Press, New York, pp 271-300.

Nakase Y 1957a Studies on Haemophilus bronchisepticus 1. The antigenic structures of $H$. bronchisepticus from a guinea pig. Kitasato Archives of Experimental Medicine 30: 57-72.

Nakase Y $1957 b$ Studies on Haemophilus bronchisepticus. II. Phase variation of $H$. bronchisepticus. Kitasato Archives of Experimental Medicine 30: 73-78.

Ross R F, Duncan J R, Switzer W P 1963 Turbinate atrophy in swine produced by pure cultures of Bordetella bronchiseptica. Veterinary Medicine 58: 566-570.

Ross R F, Switzer W P, Duncan J P 1967 Comparison of pathogenicity of various isolates of Bordetella bronchiseptica in young pigs. Canadian Journal of Comparative Medicine and Veterinary Science 31: 53-57.

Rothamsted Experimental Station, Department of Statistics 1977 Genstat: a general statistical program. Rothamsted Experimental Station, Harpenden, Herts.

Rutter J M 1981 Quantative observations on Bordetella bronchiseptica infection in atrophic rhinitis of pigs. Veterinary Record 108: 451-454.

Rutter J M 1983 Virulence of Pasteurella multocida in atrophic rhinitis of gnotobiotic pigs infected with Bordetella bronchiseptica. Research in Veterinary Science 34: 287-295.

Rutter J M, Francis L M A. Sansom B F 1982 Virulence of Bordetella bronchiseptica from pigs with or without atrophic rhinitis. Journal of Medical Microbiology 15: 105-116.

Rutter J M, Luther P D 1984 A cell culture assay for toxigenic Pasteurella multocida from atrophic rhinitis of pigs. Veterinary Record 114: 393-396.

Shimizu T, Nakagawa M, Shibata S, Suzuki K 1971 Atrophic rhinitis produced by intranasal inoculation of Bordetella bronchiseptica in hysterotomy produced, colostrum-deprived pigs. Cornell Veterinarian 61: 696-705.

Skelly B J, Pruss M, Pellegrino R, Andersen D, Abruzzo G 1980 Variation in degree of atrophic rhinitis with field isolates of Bordetella bronchiseptica. Proceedings of the 6th Congress of the International Pig Veterinary Society, held in Copenhagen, 210.

Switzer W P 1981 Bordetellosis. In: Leman AD et al. (eds) Diseases of Swine. 5th edn, Iowa State University Press, Ames, Iowa, USA, pp 497-507.

Wardlaw A C, Parton R, Hooker M J 1976 Loss of protective antigen, histamine-sensitising factor and envelope polypeptides in cultural variants of Bordetella pertussis. Journal of Medical Microbiology 9: 89-100.

Weiss A A, Falkow S 1984 Genetic analysis of phase change in Bordetella pertussis. Infection and Immunity 43: 263-269. 